\title{
Ética e investigación en Tecnología Educativa: necesidad, oportunidades y retos
}

\author{
Ethics and Research on Educational Technology: \\ needs, opportunities and challenges
}

\author{
F. Xavier Carrera Farran \\ Universitat de Lleida \\ carrera@pip.udl.cat \\ Juan González Martínez \\ Universitat Rovira i Virgili \\ juan.gonzalezm@urv.cat \\ Jordi L. Coiduras Rodríguez \\ Universitat de Lleida \\ coiduras@pip.udl.cat
}

Recibido: 13/06/2016

Aceptado: $20 / 06 / 2016$

Publicado: 30/06/2016

\section{RESUMEN}

A pesar de que la ética no ocupa un lugar privilegiado en los estudios del ámbito de la tecnología educativa, su transversalidad nos debe llevar a considerar su importancia en tres grandes ámbitos: la ética como compromiso de acción y de investigación (que parte de la cada vez más expresa recomendación institucional de respeto hacia principios éticos sólidos y compartidos), la ética como regulación del investigador (que le exige el respeto de esos principios generales en el quehacer concreto del proceso de investigación) y la ética como objeto de estudio (que entiende que las diferentes respuestas éticas a las diversas situaciones tiene un interés intrínseco que merece ser estudiado). De todo ello se reflexiona en el presente artículo, a fin de dar pautas que ayuden a promover investigaciones sobre tecnología educativa seguras, éticas y responsables.

\section{PALABRAS CLAVE}

Tecnología educativa; tecnoética; retos.

\begin{abstract}
Although ethics does not occupy a privileged place in studies in our field of educational technology, its transversality should lead us to consider its importance in three main areas: ethics as commitment to action and research (which comes from an every time more clear institutional recommendation of respecting solid and shared ethical principles), ethics as researcher processes regulator (which requires respect for these general principles in the concrete work of the research process) and ethics as an issue of study (understanding that those different ethical responses to various situations have an intrinsic interest that deserves to be studied). We reflect on all these questions in this paper, in order to provide guidelines to promote safe, ethical and responsible research on educational technology.
\end{abstract}

\section{KEYWORDS}

Educational Tecnology; Technoethics; challenges.

\section{CITA RECOMENDADA}

Carrera, F.X.; González, J.; y Coiduras, J.L. (2016). Ética e investigación en Tecnología Educativa: necesidad, oportunidades y retos. RIITE. Revista Interuniversitaria de Investigación en Tecnología Educativa, 0, 34-43. Doi: http://dx.doi.org/10.6018/riite/2016/261081 


\section{INTRODUCCIÓN}

La Declaración Nacional sobre Integridad Científica formulada recientemente por la COSCE (Confederación de Sociedades Científicas de España), la CRUE (Conferencia de Rectores de las Universidades Españolas) y el CSIC (Consejo Superior de Investigaciones Científicas) pone de manifiesto la necesidad que aún existe en nuestro país de que las instituciones dedicadas a la investigación asuman los principios éticos que deben regir la actividad científica, e impulsen e implementen sin titubeos políticas claras de integridad científica que se concreten en conductas responsables e íntegras de instituciones e investigadores. Esta apuesta es clave para incrementar la calidad científica en cualquier campo de investigación o disciplina científica y, para lograrlo, "solo una adecuada formación permitirá la pronta detección y acertada diagnosis de posibles desviaciones de las buenas prácticas científicas, así como el adecuado tratamiento y gestión de las correspondientes situaciones conflictivas" (COSCE, CRUE y CSIC, 2015, p.1).

En este contexto, la Revista Interuniversitaria de Investigación en Tecnología Educativa (RIITE), vinculada al programa de Doctorado Interuniversitario en Tecnología Educativa y al Máster Interuniversitario en Tecnología Educativa: e-Learning y Gestión del Conocimiento, surge con la intención de contribuir a la difusión de investigaciones realizadas por investigadores en formación y sus directores. Este número inicial de RIITE es, pues, una excelente ocasión para promover -entre doctorandos y estudiantes de máster y entre investigadores en activo- principios y buenas praxis de actuación en los proyectos de investigación centrados en la Tecnología Educativa.

A lo largo del artículo, queda reflejada la necesidad de abordar el componente ético en toda investigación que tenga como objeto de estudio cualquier temática o problemática vinculada con la adopción, con la innovación y con el uso de la tecnología en contextos educativos. En él también se plasman las oportunidades de mejora y los retos que supone regir todo el proceso investigador -en todos sus momentos y fases de desarrollo y en todas sus dimensiones- por principios, códigos y pautas éticas.

Para los investigadores en formación, el contenido del artículo les ha de ayudar, sin duda, a adoptar un compromiso personal con la necesidad de desplegar siempre una investigación rigurosa, crítica, responsable y ética. A los investigadores en activo, por su parte, les resultará útil para supervisar su quehacer investigador y contrastar hasta qué punto sus planteamientos y sus formas de trabajo están en consonancia con las prácticas éticas de referencia. A unos y otros también les puede aproximar a nuevos escenarios y a nuevos problemas derivados de la presencia de las tecnologías digitales en la educación que den pie a investigaciones originales y relevantes.

Para ello, el artículo se acerca a la ética en la investigación en Tecnología Educativa desde una triple vertiente: la ética como compromiso de investigación, la ética como regulación de la investigación y la ética como objeto de investigación. En la ética como compromiso nos aproximamos a los principios y a las directrices que deben regir nuestras investigaciones. En cuanto a la ética como regulación, mostramos qué debe tenerse en cuenta durante la investigación en relación con los investigadores, con los participantes, con el proceso desplegado y con la divulgación. Y sobre la ética como objeto de estudio, planteamos nuevas problemáticas y nuevos espacios de investigación centrados en las relaciones surgidas del binomio tecnología y educación en un contexto social diverso y complejo.

\section{LA ÉTICA COMO COMPROMISO DE INVESTIGACIÓN}

Igual que no existe una tecnología neutra (Buchanan, 1965; Pacey, 1983) tampoco podemos hablar de neutralidad en la investigación llevada a cabo en Tecnología Educativa. Todo lo contrario. El desarrollo de cualquier investigación está permanentemente condicionado por la continua y constante toma de decisiones por parte del equipo de investigación y de cada uno de sus integrantes. Se trata de una cuestión de elecciones que, tomadas conscientemente o no, van a condicionar la investigación en una dirección y con una intensidad ética determinadas. Así pues, desde la temática sobre la que decidimos investigar hasta las vías escogidas para la divulgación de los resultados pasando por la definición de las preguntas, de los objetivos y de las hipótesis; por el paradigma adoptado y por el tipo de 
diseño elaborado; por la selección de participantes y muestras; por los instrumentos utilizados en la recogida de datos; enpor los criterios de análisis adoptados y su aplicación y por la discusión de los resultados obtenidos -entre otras actuaciones-, todo ello va a estar condicionado por las decisiones que vayamos adoptando; y estas, a su vez, por los principios y valores que las rijan.

En este sentido, los principios de respecto por las personas, justicia y beneficencia ${ }^{1}$ (no maleficencia) establecidos en el Informe Belmont (DHEW, 1979) fueron un punto de arranque en el intento de resolver los problemas y conflictos éticos que se dan en las investigaciones llevadas a cabo con personas. Más adelante, en la segunda Conferencia Mundial sobre Integridad en la Investigación, se discutieron y debatieron principios y responsabilidades que deben considerarse a nivel mundial para fomentar una mayor integridad de la investigación realizada en todo el mundo y en todos los ámbitos. En esta Conferencia se aprobó, sin ánimo regulador y desvinculado de las políticas oficiales de países y entidades organizadoras, la Singapore Statement on Research Integrity (World Conference on Research Integrity, 2010). En la declaración se establecen cuatro principios básicos que deben sustentar la integridad de la investigación: (1) honestidad en todos los aspectos de la investigación; (2) responsabilidad en la ejecución de la investigación, (3) cortesía profesional e imparcialidad en las relaciones laborales y (4) buena gestión de la investigación en nombre de otros.

Entidades y organizaciones como la British Educational Research Association (BERA), la American Educational Research Association (AERA), la European Science Foundation (ESF), la All European Academies (ALLEA) o el Economic and Social Research Council (ESRC) -entre otras que ya venían trabajando en promover investigaciones éticas y responsables- hacen propia la necesidad de mejorar la integridad en la investigación social y educativa. Para ello, establecen directrices y códigos específicos donde se recogen pautas de conducta y de buenas prácticas partiendo de principios éticos específicos que sintetizamos a continuación. Por su parte, BERA (2011) establece que toda investigación educativa debe llevarse a cabo dentro de una ética de respeto a la persona, al conocimiento, a los valores democráticos, a la calidad de la investigación para la educación y a la libertad académica. Para AERA (2011), los principios esenciales que deben considerar los investigadores en cualquier contexto educativo son: competencia profesional, integridad, responsabilidad (profesional, científica y académica), respeto (por los derechos, por la dignidad y por la diversidad de las personas) y responsabilidad social. ESF y ALLEA (2011) acuerdan como principios que deben regir el código de conducta en la investigación científica y académica: la honestidad en la comunicación; la fiabilidad en la realización de la investigación; la objetividad; la imparcialidad y la independencia; la apertura y accesibilidad; el cuidado; la equidad y la responsabilidad con los científicos e investigadores del futuro. Por último, la versión de 2012 actualizada del ESRC (2015), define seis principios fundamentales de la investigación ética: un primero que aglutina los de integridad, calidad y transparencia; la participación voluntaria; la utilidad y el beneficio (por encima del riesgo); la información exhaustiva a los participantes; la confidencialidad y el anonimato de los participantes y, un último principio, de independencia de la investigación.

Todos y cada uno de dichos principios deben guiar cualquier investigación educativa llevada a cabo independientemente de su finalidad, sus participantes, su duración, su coste, el organismo financiador o su impacto. De ellos, consideramos esenciales los principios de respecto, honestidad, responsabilidad, integridad, imparcialidad, transparencia, confidencialidad, independencia y voluntariedad.

La consideración de tales principios de modo que guíe toda la actividad de investigación desarrollada denota el compromiso ético del investigador. Un compromiso de acción que requiere -de acuerdo con las tesis de Jonas (1995), que compartimos- adoptar la responsabilidad como el principio rector de toda acción tecnológica con independencia del ámbito y de la especialidad en que se dé.

1 Beneficencia entendida como obligación moral de actuar a favor y en ayuda de otros, de modo que se contribuya a promover sus beneficios y legítimos intereses. 
Estamos, por tanto, ante un principio necesario, aunque no suficiente, en las investigaciones centradas en la educación y la tecnología en tanto que (Olcott, Carrera, Gallardo y González, 2015):

a. El sentimiento de responsabilidad personal va más allá de la responsabilidad formal y legal, a la vez que enlaza con otros valores y principios morales que también deben adoptarse en la investigación educativa.

b. Uno es responsable de sus actos y de las consecuencias de sus actos.

c. Antes de actuar uno siempre debe anticiparse y prever los efectos de las acciones que se desean llevar a cabo.

\section{LA ÉTICA COMO REGULACIÓN DE LA INVESTIGACIÓN}

Un paso más allá en las consideraciones anteriores nos lleva a plantearnos que la adopción de valores y principios rectores en la actividad investigadora no debe -ni puede- quedar reducida a una mera declaración inicial de intenciones a modo de marco de actuación responsable. Sin duda, el compromiso ético debe impregnar todas las fases y todas las actuaciones que se den antes, durante y después de la investigación. Como concreción de ello, en los siguientes subapartados recogemos las principales pautas de actuación ética según estén focalizadas en los investigadores, en las personas investigadas, en el proceso de investigación o en la propiedad intelectual y en la divulgación de resultados y conclusiones. Esta explicación debe entenderse como una primera aproximación, condicionada por las limitaciones de espacio; la consulta de los códigos y de las declaraciones citados en este artículo permitirá a cualquier investigador profundizar en cómo proceder correctamente en el desarrollo ético de sus estudios científicos.

\subsection{Acerca de los investigadores}

Competencias y limitaciones. El investigador debe conocer y reconocer sus competencias y sus limitaciones como investigador y debe actuar en consecuencia sin poner en riesgo la calidad y el rigor de la investigación. Al igual que los investigadores expertos, los investigadores noveles y los investigadores en formación incrementarán su competencia investigadora trabajando en equipo con otros investigadores y/o contrastando con colegas y expertos los procedimientos seguidos.

Conflicto de intereses. Todo investigador debe reconocer aquellas situaciones que le suponen un conflicto personal de intereses motivadas por cuestiones personales, profesionales económicas o de otra índole o bien por cualquier otra causa o circunstancia. En consecuencia, debe renunciar a participar en la investigación de forma directa o indirecta o bien -atendiendo al alcance del conflicto- a explicitar con exactitud la potencial situación de conflicto de intereses. Esta renuncia debe también efectuarse cuando el investigador participe en actividades de evaluación de proyectos, de artículos en publicaciones o de comunicaciones en reuniones científicas en que se dé el conflicto de intereses.

Confidencialidad de participantes y de datos. Cualquier investigador debe mantener, durante toda la investigación y en la divulgación y diseminación de los resultados, la confidencialidad acerca de cualquier información que afecte a los participantes o que permita su identificación y de los datos obtenidos. Debe velarse por la privacidad de los datos en cualquier soporte (digital o analógico) y registro (escrito, sonoro, visual, audiovisual) en que se encuentren.

Continuidad de la investigación. El investigador debe realizar sus estudios sin poner en peligro o riesgo investigaciones futuras que puedan realizarse en el mismo contexto, en una misma temática o con los mismos participantes.

Relegar conductas fraudulentas. El investigador nunca debe llevar a cabo -conscientemente o por negligencia- actuaciones engañosas o dolosas como puedan ser, entre otras: investigar con una finalidad 
ilícita o perjudicial para los participantes o para terceros; inventar, falsificar o tergiversar datos, hallazgos o conclusiones; plagiar o apropiarse de los resultados de otros investigadores.

\subsection{Acerca de los investigados}

Consentimiento informado. Las personas, los grupos y las organizaciones deben expresar su consentimiento informado antes de iniciarse la investigación en la que van a participar. Dicho consentimiento refleja que entienden en qué consiste su participación, que están de acuerdo con ella y que acceden libremente y sin ningún tipo de coacción. El investigador deberá informar asimismo del derecho que asiste al participante de retirarse de la investigación y también de que se le informará de cualquier cambio de orientación que se dé en la investigación. En aquellos estudios en que sea necesario no informarles antes del inicio de la investigación (atendiendo al objeto y objetivos de la investigación) se buscará la aprobación previa institucional y el consentimiento personal de los participantes tras finalizar la investigación.

Privacidad y anonimato. Los participantes tienen derecho a que sus datos personales y los derivados de su actividad en la investigación sean confidenciales y se mantengan en el anonimato a menos que haya por su parte una renuncia expresa, específica y voluntaria, en soporte escrito. Por el contrario, los investigadores deben reconocer el derecho de los participantes a ser identificados en cualquier publicación derivada de la investigación si así lo desea y si así se considera. Además, los investigadores deben cumplir con la legislación vigente relativa a la protección de datos en el país en que se lleva a cabo el estudio.

Colectivos vulnerables. El investigador prestará especial atención cuando en su trabajo se vean involucrados colectivos potencialmente vulnerables como pueden ser los niños, los adolescentes y los jóvenes; las personas con dificultades de aprendizaje o con deterioro cognitivo o las que carezcan de autonomía para tomar decisiones por sí mismas. En dichos casos, extremará sus actuaciones a fin de preservar y garantizar todos sus derechos.

Derecho al abandono. Los participantes tienen derecho a retirarse en cualquier momento y por cualquier razón de la investigación sin tener que justificar su decisión. Los investigadores, a su vez, deben informar siempre a los participantes de este derecho.

Participación no discriminatoria. Salvo que existan razones metodológicas o de finalidad del estudio que lo justifiquen, los participantes deben incorporarse a la investigación sin ser segregados por motivos de género, categoría social, grupo étnico, orientación sexual, religión, capacidades... u otros factores que supongan una diferenciación personal o colectiva no respetuosa con la diversidad, dignidad y derechos de los participantes.

\subsection{Sobre el proceso de investigación}

Interés científico y social. Cualquier investigación debe responder al interés científico y social por encima de cualquier otro de particular, sea este individual o colectivo. Los investigadores deben sopesar los beneficios que puede aportar su trabajo respecto de los riesgos que pueda comportar su realización.

Adecuación metodológica. Los investigadores adoptarán métodos, instrumentos y procedimientos que respondan al diseño y a los objetivos de la investigación y los aplicarán de manera que: (a) permitan el logro de resultados objetivos, válidos y fiables y (b) garanticen los derechos de los participantes y de las instituciones colaboradoras.

Transparencia y objetividad. Toda la actividad investigadora debe ceñirse a una actuación objetiva y transparente que garantice la solvencia y la credibilidad de los resultados obtenidos y facilite contrastarlos en la medida que el diseño y desarrollo de la investigación y la confidencialidad de los datos lo permitan.

Disponibilidad y acceso a datos. Los investigadores deben compartir con otros investigadores los datos y los resultados obtenidos al finalizar la investigación cuando estos deseen replicar o dar continuidad al estudio, siempre sin contravenir los compromisos de confidencialidad acordados con los participantes y con terceros. Para ello, deberán almacenar los datos primarios y secundarios de manera 
segura y accesible. Asimismo, divulgaran por las vías de diseminación científica habituales dichos resultados a la mayor brevedad de tiempo que les sea posible. Al mismo tiempo deben velar por la custodia de dichos datos sin cederlos a terceros que puedan obtener beneficios de su acceso y uso.

Minimización del impacto. El investigador siempre optará por estrategias y uso de técnicas que no tengan consecuencias personales o sociales negativas. En aquellos estudios cuyo diseño otorgue algún tipo de ventaja, real o presunta, a una parte de los participantes y deje sin el beneficio derivado de dicha aplicación al resto, el investigador ofrecerá iniciativas posteriores a los participantes afectados que permitan compensar el posible perjuicio ocasionado.

Corrección del error. En parte, puede entenderse como una concreción de lo anterior; en todo caso, y en la medida en que nuestra investigación detecte disfunciones o aspectos negativos (generados directamente por nosotros, o no), debemos colaborar en su resolución (sea interviniendo nosotros mismos para resolverlos, sea poniéndolos en conocimiento de las instancias competentes para ello).

\subsection{Sobre la propiedad intelectual y la divulgación}

Informe de investigación, artículos, comunicaciones y otros formatos de divulgación. Los investigadores deben publicar y difundir los resultados y conclusiones de sus investigaciones a la mayor brevedad posible desde su finalización. Sus publicaciones deben ser honestas, transparentes y precisas.

Autoría. Debe reconocerse la identidad de los autores de la investigación, independientemente de las fuentes de financiación que también deben hacerse públicas. Todo autor debe expresar su consentimiento a figurar como tal y todos los autores son responsables del contenido de la publicación a menos que se indique explícitamente lo contrario. El orden de la autoría vendrá marcado por criterios de dirección y contribución creativa durante la investigación y en la participación en la elaboración del documento de divulgación.

Colaboradores y participantes. En la medida en que los informantes y los colaboradores nos facilitan con su participación los datos que necesitamos para llevar a cabo nuestra investigación, debemos corresponder a su generosidad poniendo a su alcance las conclusiones a las que lleguemos gracias a ellos. Esto puede realizarse por medio de una petición expresa en que sondeemos su voluntad de ser mantenidos al corriente, o bien de manera genérica, garantizando que las líneas generales de las conclusiones serán accesibles para el público en general. En las publicaciones debe reconocerse, con su consentimiento, el trabajo y la contribución realizada a la investigación por parte de los colaboradores. Y también debe figurar el reconocimiento a la participación de las personas investigadas.

Contenido de la publicación. El contenido de la publicación debe ser objetivo y estar libre de juicios intencionados. Debe hacerse una correcta referenciación de fuentes directas e indirectas de acuerdo con el estándar adoptado. En ningún caso debe plagiarse el contenido de otras obras, estudios y autores y, en el caso de que el contenido sea similar al de otros artículos debe expresarse esta circunstancia.

Revisión y evaluación. Editores y revisores deben rechazar participar en la evaluación o publicación de artículos y comunicaciones cuando entren en conflicto de intereses con la obra, el contenido o sus autores. Los revisores, además de mantener la confidencialidad sobre el manuscrito y sus autores, deben efectuar evaluaciones objetivas, precisas, detalladas y argumentadas. Tampoco deben utilizar datos ni parte del contenido sin el permiso expreso de los autores.

\section{LA ÉTICA COMO OBJETO DE INVESTIGACIÓN}

Es difícil renunciar a aplicar las pautas y orientaciones recogidas en el apartado anterior si deseamos mejorar la calidad de la investigación educativa en general, y de la Tecnología Educativa en particular. Sin embargo, otra línea que permite ahondar en esta mejora radica en ampliar el objeto y las temáticas de las investigaciones que se dan en este ámbito. 
Tradicionalmente, se tiende a investigar sobre los procesos de integración y utilización en contextos educativos de herramientas, dispositivos, sistemas, programas, aplicaciones, recursos...; sobre las competencias digitales de los profesionales de la formación y de la educación en contextos formales, no formales e informales; sobre los procesos cognitivos asociados a la integración de la tecnología en el aprendizaje; sobre la naturaleza del propio conocimiento en una sociedad cada vez más digital, etc.; pero son muchas menos las investigaciones que parten de dilemas y problemas relacionados con cuestiones éticas que afectan a la presencia y al uso de las tecnologías digitales en educación o aquellas en las que los valores cobran un protagonismo central. Como consecuencia de ello, estamos ante un déficit importante de investigación en este triángulo delimitado por la ética, la tecnología y la educación que abre un sinfín de posibilidades a los investigadores que se sientan atraídos por una parcela de conocimiento con muchísimas -e interesantísimas- posibilidades por explorar.

Siguiendo en parte el modelo esbozado por Olcott (2002) para la toma de decisiones en el ámbito de la tecnoética (entendida como ética de la tecnología) sugerimos a continuación una relación incompleta de posibles preguntas que apuntan a la investigación en diferentes puntos de la intersección entre los aspectos educativos, los tecnológicos y los éticos. A fin de clarificar el discurso, las diferenciamos, según la temática y según el ámbito de su impacto, entre cuestiones filosóficas, cuestiones educativas, cuestiones socio-personales y cuestiones económicas. Algunas de las cuestiones, excesivamente genéricas o extensas, pueden dar pie a supuestos de investigación más específicos. Otras, en cambio, por su alcance más reducido, pueden ser un preámbulo que dé lugar a investigaciones concretas. En cualquier caso, todas ellas son cuestiones abiertas, pensadas para abrir nuevos espacios de investigación en Tecnología Educativa.

\section{Cuestiones filosóficas}

¿Cómo ha evolucionado el pensamiento ético acerca de la tecnología a lo largo de la historia?

Incorporar las tecnologías digitales a la educación ¿no supone, de facto, una contribución a la homogenización cultural y a la pérdida de la identidad cultural de colectividades y comunidades locales?

¿Se puede construir una pedagogía crítica de las tecnologías digitales en la educación desde la praxis educativa?

¿Hasta qué punto la incorporación de robots en los procesos educativos puede suponer la despersonalización de la educación?

¿Qué límites deben considerarse en la incorporación de las tecnologías digitales en la educación?

\section{Cuestiones educativas}

¿Hasta qué punto las tecnologías digitales son sinónimo de calidad educativa y mejoran la calidad de la educación?

¿Cómo afecta el plagio académico, facilitado por las tecnologías digitales, en el desarrollo moral de los estudiantes?

¿Cómo deberían ser las metodologías de aprendizaje en red para que realmente sean inclusivas, participativas y democráticas?

Los nuevos escenarios de formación que genera la innovación tecnológica ¿provocan realmente una transformación profunda y beneficiosa para el aprendizaje y las personas o no son más que otro objeto de consumo tecnológico?

¿Cómo afecta la calidad de la información y de los contenidos en red al desarrollo personal y al aprendizaje escolar de los niños y jóvenes?

¿Qué presencia tiene la ética en los planes de integración de las TIC y en los proyectos educativos con tecnología que se llevan a cabo en los centros escolares? 
¿El análisis, la evaluación y la valoración de situaciones educativas en que interviene la tecnología bajo qué modelos educativos y principios y códigos éticos se lleva a cabo?

\section{Cuestiones socio-personales}

¿Cómo contribuye la escuela a la configuración de la identidad digital de los niños y adolescentes?

¿Se respetan o se vulneran los derechos individuales de los estudiantes en los entornos de formación en red?

¿Contribuyen las tecnologías al desarrollo de la competencia ética de las personas?

¿Hasta qué punto las redes sociales son una forma de invasión de la privacidad y la intimidad de las personas que condiciona su desarrollo personal y social?

¿La formación orientada a forjar desde la escuela productores y consumidores de información contribuye a una ciudadanía más crítica y libre?

¿En que medida las tecnologías digitales contribuyen al desarrollo de la participación y de la ciudadanía o a la alienación y a la reproducción de contravalores?

\section{Cuestiones económicas}

¿Las tecnologías digitales favorecen una visión mercantilista de la educación que va a transformar los sistemas educativos nacionales, especialmente en la formación universitaria?

¿Qué impactos genera en los lugares de trabajo la inclusión de las tecnologías en la formación?

¿Qué presencia tienen los valores en las políticas y decisiones educativas tomadas por los gobiernos nacionales, regionales y locales y por los organismos internacionales?

¿Las tecnologías digitales son una vía para la exclusión o para la inclusión social?

¿Hasta qué punto los factores económicos frenan el despliegue y la plena integración de las tecnologías en las escuelas?

¿Qué impacto laboral y económico va a tener en las próximas décadas la deficiente formación en competencias digitales que reciben buena parte de los escolares en la actualidad?

\section{A MODO DE CONCLUSIONES}

A lo largo del artículo hemos intentado aislar, en la medida de lo posible, las tres grandes esferas de conocimiento que subyacen de la intersección de la tecnología, la educación y la ética. $\mathrm{Y}$, lejos de ir cerrando esos asuntos, hemos tratado precisamente de ofrecer una perspectiva nítida de cuán largo e interesante es el camino que queda por recorrer en cada una de esas esferas, cumpliendo con el objetivo de identificar oportunidades y retos que debemos asumir en nuestro ámbito.

En este sentido, y por cuanto la ética impregna cualquier acción humana, reafirmamos nuestra convicción de que no hay ninguna investigación neutra. No es suficiente con incorporar una reflexión general que se traduzca solamente en un compromiso de la investigación -y de los investigadores- con un planteamiento ético de su quehacer profesional. Dicho compromiso debe ser auténtico y profundo hasta el punto de que debe guiar y materializarse en todas y cada una de las acciones y decisiones que se producen en el desarrollo de la investigación. Ahí radican, a nuestro modo de ver, la transversalidad de la ética y la necesidad de impregnar de contenido ético cada una de las investigaciones que planteamos en el ámbito de la tecnología educativa: usar la tecnología tiene implicaciones éticas; investigar sobre ello, por supuesto, también. 
Con todo, no queda ahí la cuestión, puesto que los nuevos escenarios, las nuevas relaciones del ser humano frente al propio aprendizaje y en su relación con la Sociedad del Conocimiento también tienen connotaciones éticas -al fin y al cabo, lo humano siempre las tiene- que pueden constituir un interesante y aún, en nuestro contexto, poco explotado ámbito de estudio. No hay duda de que en la medida en que no hay educación, o tecnología educativa, o investigación en tecnología educativa, sin ética -buena o mala-, todo ello puede y debe ser motivo de nuestro interés como investigadores.

Por tanto, en directa relación con el título del artículo, la ética -o, si se quiere, el triángulo formado por la ética, la educación y la tecnología- nos plantea retos que no podemos no asumir, oportunidades que no deberíamos desaprovechar y necesidades que debemos atender con responsabilidad y compromiso.

\section{REFERENCIAS BIBLIOGRÁFICAS}

AERA (2011). Code of Ethics. Educational Researcher, 40, 3, 45-156. DOI: 10.3102/0013189X11410403

Buchanan, R. A. (1965). Technology and Social Progress. Oxford: Pergamon Press.

BERA (2011). Ethical Guidelines for Educational Research. Recuperado de: https://www.bera.ac.uk/wpcontent/uploads/2014/02/BERA-Ethical-Guidelines-2011.pdf?noredirect=1

COSCE, CRUE y CSIC (2015). Declaración Nacional sobre Integridad Científica. Recuperado de: http://www.csic.es/etica-en-la-investigacion

DHEW (1979). The Belmont Report: Ethical Principles and Guidelines for the Protection of Human Subjects of Research. Washington, DC: Department of Health, Education, and Welfare. Recuperado de: http://www.hhs.gov/ohrp/regulations-and-policy/belmont-report/index.html

ESF y ALLEA (2011). The European Code of Conduct for Research Integrity. Recuperado de: http://www.esf.org/fileadmin/Public_documents/Publications/Code_Conduct_Researchlntegrity.pdf

ESRC (2015). Framework for Research Ethics. Recuperado de: http://www.esrc.ac.uk/files/funding/guidance-for-applicants/esrc-framework-for-research-ethics-2015/

Jonas, H. (1995). El principio de responsabilidad. Ensayo de una ética para la civilización tecnológica. Barcelona: Herder.

Olcott, D. (2002). Ética y tecnología: desafíos y elecciones inteligentes en una sociedad tecnoética. En D.E., Hanna (Coord.), La enseñanza universitaria en la era digital. Barcelona: Octaedro-EUB

Olcott, D., Carrera, X., Gallardo, E. y González, J. (2015). Ética y Educación en la era digital: perspectivas globales y estrategias para la transformación local en Cataluña. RUSC. Universities and Knowledge Society Journal, 12(2), 59-72, DOI: http://dx.doi.org/10.7238/rusc.v12i2.2455

Pacey, A. (1983). La cultura de la tecnología. México: Fondo de Cultura Económica.

World Conference on Research Integrity (2010). Singapore Statement on Research Integrity. Recuperado de: http://www.singaporestatement.org/statement.html 


\title{
INFORMACIÓN SOBRE LOS AUTORES
}

\author{
F Xavier Carrera Farran \\ Profesor Titular de Universidad \\ Departamento de Pedagogía y Psicología - Universidad de Lleida
}

Doctor en Psicopedagogía. Premio extraordinario de Doctorado. Coordinador en la Universidad de Lleida del Programa de Doctorado Interuniversitario en Tecnología Educativa. Miembro del grupo de investigación COMPETECS (Competencias, Tecnología, Educación y Sociedad) de la Universidad de Lleida [2014 SGR 1611]. Sus líneas de investigación se centran en la Tecnología Educativa; la aplicación de metodologías activas en educación y en la formación basada en competencias.

http://webgrec.udl.es/webpages/personal/cat/000071_carrera.pip.udl.cat.html

\section{Juan González Martínez}

Departament de Pedagogia. Universitat Rovira i Virgili

Profesor del Departament de Pedagogia de la Universitat Rovira. Doctor en Filología Hispánica (UNED) y en Tecnología Educativa (URV): Coordinador del Máster en Tecnología Educativa: e-Learning y Gestión del Conocimiento de la URV. Miembro del grupo de investigación consolidado ARGET (Applied Research Group in Education and Technology), con identificador 2014SGR1399. Investigador del proyecto Simul@b. Laboratorio de simulaciones 3d para el desarrollo de la competencia digital docente, financiado por el Ministerio de Economía y Competitividad (ref. EDU2013-42223-P). Su principal línea de investigación tiene que ver con la competencia digital (concepto e instrumentos para su medición en los niveles educativos de Secundaria y Universidad) y la competencia digital docente.

\section{Jordi L. Coiduras Rodríguez}

Profesor agregado Departamento de Pedagogía y Piscología - Universidad de Lleida

Doctor en Psicopedagogía. Centra su actividad de investigación en la tecnología educativa y en la formación dual. Es docente en los grados de educación infantil, de educación primaria y en el máster interuniversitario "e-learning y gestión del conocimiento". Actualmente coordina el G. de E. Primaria en modalidad dual. Es investigador del grupo COMPETECS (Competencias, Tecnología, Educación y Sociedad) de la Universidad de Lleida [2014 SGR 1611]. http://webgrec.udl.es/webpages/personal/cat/000135_coiduras.pip.udl.cat.html

\section{$(\mathrm{cc}) \mathrm{BY}_{\mathrm{B}-\mathrm{NC}}$}

Los textos publicados en esta revista están sujetos a una licencia de Reconocimiento 4.0 España de Creative Commons. Puede copiarlos, distribuirlos, comunicarlos públicamente y hacer obras derivadas siempre que reconozca los créditos de las obras (autoría, nombre de la revista, institución editora) de la manera especificada por los autores o por la revista. La licencia completa se puede consultar en:Licencia Creative Commons Atribución-NoComercial 4.0 Internacional. 